\title{
Habilidades gerenciales en estudiantes de Administración de Negocios de una universidad privada de Lima, 2020
}

\author{
Managerial skills in Business Administration students of a Private University of Lima, 2020 \\ Habilidades de gestão em estudantes de Administração de Empresas em uma Universidade \\ Privada em Lima, 2020
}

\section{ARTÍCULO DE INVESTIGACIÓN}

\author{
Layo Cárdenas Oscco \\ ccardenasos@ucvvirtual.edu.pe \\ https://orcid.org/0000-0001-8719-0705 \\ Universidad César Vallejo, Trujillo-Perú
}

\author{
Lidia Neyra Huamani \\ lneyrah@ucvvirtual.edu.pe \\ https://orcid.org/0000-0001-6261-2190 \\ Universidad César Vallejo, Trujillo-Perú
}

Recibido 6 de mayo 2021 | Arbitrado y aceptado 15 de junio 2021 | Publicado en 01 julio 2021

\section{RESUMEN}

El objetivo del presente estudio es determinar el nivel de habilidades gerenciales en estudiantes de Administración de Negocios de una universidad privada de Lima, 2020. Desde la perspectiva metodológica, la presente investigación se realiza con el enfoque cuantitativo. El tipo de investigación es básica con diseño descriptivo. La muestra fue aleatoria y se consideró a 210 estudiantes de la Facultad de Administración y Negocios. Se empleó el instrumento estandarizado de 31 ítems. Los datos recolectados fueron analizados mediante el programa estadístico SPSS. Resultados: predominio del nivel bueno con 56,7 \%. Es decir, 119 de 210 se encuentran en este nivel. Seguido del nivel regular con 87 estudiantes que representa $41,4 \%$. Luego, el nivel deficiente con 4 estudiantes que representa $1,9 \%$. Conclusión, se ha evidenciado que la mayoría de los estudiantes de Administración se encuentra en un nivel bueno de habilidades gerenciales.

Palabras clave: Habilidad; gerencial; técnicas; conceptual

\section{ABSTRACT}

The objective of this study is to determine the level of managerial skills in Business Administration students at a private university in Lima, 2020. From the methodological perspective, this research is carried out with a quantitative approach. The type of research is basic with a descriptive design. The sample was random and 210 students from the Faculty of Business and Administration were considered. The standardised 31item instrument was used. The data collected were analysed using SPSS statistical software. Results: $56.7 \%$ of the students were at the good level. That is, 119 out of 210 students were at this level. This is followed by the average level with 87 students, which represents $41.4 \%$. Then, the poor level with 4 students representing 1.9\%. In conclusion, it is evident that the majority of Management students are at a good level of management skills.

Key words: Ability; managerial; techniques; conceptual

\section{RESUMO}

O objectivo do presente estudo é determinar o nível de competências de gestão em estudantes de Administração de Empresas de uma universidade privada em Lima, 2020. Do ponto de vista metodológico, a presente investigação é conduzida com a abordagem quantitativa. O tipo de investigação é básico com desenho descritivo. A amostra foi aleatória e foram considerados 210 estudantes da Faculdade de Negócios e Administração. Foi utilizado o instrumento padronizado de 31 artigos. Os dados recolhidos foram analisados utilizando o programa estatístico SPSS. Resultados: predominância do bom nível com 56,7\%. Ou seja, 119 dos 210 estudantes encontravam-se a este nível. Seguido pelo nível regular com 87 estudantes representando 41,4\%. Depois, o nível deficiente com 4 estudantes representando $1,9 \%$. Conclusão, foi evidenciado que a maioria dos estudantes de Gestão está num bom nível de competências de gestão.

Palavras-chave: Habilidade; gestão; técnicas; conceptuais 


\section{INTRODUCCIÓN}

Las habilidades gerenciales son capacidades y conocimientos que todo administrador debe tener dominio. Los cuales, deben ser desarrolladas por el emprendedor para realizar las actividades de administración, en el rol del Gerente o Director de una organización (Koontz et al., 2012).

En este sentido, los gerentes de hoy se ven enfrentados a muchos retos, los cuales les exigen desarrollar las habilidades gerenciales suficientes y necesarias a fin de responder de manera acertada, eficiente y pertinente a los mismos (Naranjo y González, 2012).

En este contexto, es necesario que el gerente logre manejar sus emociones y consiga la sinergia de las diferentes habilidades gerenciales, para lograr la integración interna y el compromiso de los colaboradores (Carhuancho et al., 2019). Además se han demostrado que las habilidades gerenciales son un factor interno de las Pymes que influyen considerablemente en su competitividad empresarial (Leyva-Cárdenas et al., 2017).

Asimismo, son consideradas como el conjunto de conocimientos, habilidades, comportamientos $\mathrm{y}$ actitudes que una persona debe poseer para ser efectiva en un amplio abanico de puestos y en distintas clases de organizaciones (Hellriegel et al., 2017).

Es evidente la necesidad de las habilidades gerenciales. Las cuales son determinantes para el éxito de la organización y advierten que las destrezas directivas son como un vehículo a través del cual la estrategia y la práctica de herramientas administrativas llegan a conseguir el resultado esperado (Whetten y Cameron, 2005).

Los estudiantes de Educación superior universitaria de las carreras profesionales de Administración deben desarrollar las habilidades gerenciales. Puesto que, el valor de la estrategia en una competencia proviene del desarrollo de la habilidad gerencial para intervenir en un sistema complejo con solo cierta información limitada y con ello producir un cambio predecible y deseable en el equilibrio del sistema. Al contar con habilidades gerenciales será posible crear una planeación estratégica adecuada en las Pymes que ayude a la gerencia a determinar las acciones que deberá llevar a cabo para lograr el objetivo o la meta de la organización (Leyva-Cárdenas et al., 2017).

En este sentido, las habilidades gerenciales son el vehículo mediante el cual la estrategia y la práctica de la administración, las herramientas y las técnicas, los atributos de la personalidad y el estilo trabajan para producir resultados eficaces dentro de administración de las organizaciones (Whetten y Cameron, 2011).

Asimismo, se han demostrado que la combinación de educación, experiencia y habilidades personales genera unas habilidades gerenciales únicas que empoderan a las mujeres sujeto de estudio (Cassab y Mayorca, 2018).

Además, la efectividad externa o rendimiento de la empresa implica que la organización logre sus objetivos de producción, mercado e imagen, de tal forma que pueda sostenerse y desarrollarse en el tiempo; para ello es indispensable que quien toma las decisiones posea ciertas capacidades y conocimientos que pueda aplicar en la empresa (González y Ley, 2019).

El dominio de las habilidades gerenciales es una necesidad en los estudiantes de Administración. Por ello, se debe priorizar en todo el proceso de formación profesional. Puesto que el desarrollo de las habilidades gerenciales les permitirá tomar decisiones gerenciales en el campo laboral. En este sentido, nuestro objetivo de investigación fue determinar el nivel habilidades gerenciales en estudiantes de Administración de Negocios de una Universidad Privada de Lima, 2020.

Se han demostrado que las habilidades gerenciales para una cultura de la innovación deja interpretar que en las organizaciones universitarias 
estudiadas no se fusionan nuevos valores de gestión con los valores académicos tradicionales, de manera que todos los niveles de la institución trabajen para mejorar la cultura académica (Donawa y Gámez, 2019).

\section{MÉTODO}

El presente estudio es cuantitativo que se circunscribe dentro del paradigma positivista. El tipo de investigación es básica y de diseño descriptivo. Este tipo de estudios descriptivos tienen como finalidad especificar propiedades y características de conceptos, fenómenos, variables o hechos en un contexto determinado (Hernández y Mendoza, 2018). En el estudio se describe el nivel de habilidades gerenciales en los estudiantes de Administración de Negocios de una Universidad Privada de Lima.
Se recogió la data a través la técnica de encuesta. El instrumento fue un cuestionario estandarizado del año 2019. El cual, estuvo centrado en las dimensiones: Habilidades técnicas, Habilidades de trato del personal administrativo y Habilidades conceptuales (Carhuancho et al., 2019). El instrumento presenta una alta confiabilidad de 0.968. Asimismo, la escala de medición es como sigue: nunca (1), casi nunca (2), a veces (3), casi siempre (4) y siempre (5). Este cuestionario de 31 preguntas fue respondido en Google form por los 210 estudiantes desde setiembre hasta diciembre del 2020. Luego se han procesado los datos con el programa estadístico SPSS. (Tabla 1).

Tabla 1. Descripción de dimensiones e indicadores.

\begin{tabular}{lll}
\hline \multicolumn{1}{c}{ Dimensiones } & \multicolumn{1}{c}{ Indicadores } & \multicolumn{1}{c}{ Ítems } \\
\hline Habilidades técnicas & Software & $1,2,3,4$ \\
& Idioma & 5,6 \\
& Negociación y decisiones & $7,8,9,10$ \\
Habilidades de trato personal & Autocontrol & $11,12,13,14$ \\
& Autoconfianza & 15,16 \\
& Equilibrio emocional & 17,18 \\
& Empatía & $19,20,21$ \\
Habilidades conceptuales & Desarrollo de nuevos conceptos & $22,23,24,25,26$ \\
& Resolución de problemas & $27,28,29,30,31$ \\
\hline
\end{tabular}

Fuente: (Carhuancho et al., 2019). 
La muestra seleccionada de forma aleatoria fueron 210 estudiantes matriculados en el semestre académico 2020-II de la Facultad de Administración de Negocios de una universidad privada de Lima. De los cuales, 90 fueron de sexo masculino que representa $43 \%$ y 120 de sexo femenino que representa el $57 \%$.

\section{RESULTADOS Y DISCUSIÓN}

\section{Habilidades gerenciales}

En la Tabla 2 la variable habilidades gerenciales, de 210 estudiantes que representan el 100\% se evidencia que 4 estudiantes que representa el 1,9
\% se encuentra en el nivel deficiente. Asimismo, 87 estudiantes que representa $41,4 \%$ se encuentran en un nivel regular. También 119 estudiantes que representa $56,7 \%$ se encuentran en un nivel bueno.

Tabla 2. Habilidades Gerenciales.

\begin{tabular}{llrrrr}
\hline & Frecuencia & Porcentaje & Porcentaje válido & Porcentaje acumulado \\
\hline Válido & Deficiente & 4 & 1,9 & 1,9 & 1,9 \\
& Regular & 87 & 41,4 & 41,4 & 43,3 \\
& Buena & 119 & 56,7 & 56,7 & 100,0 \\
& Total & $\mathbf{2 1 0}$ & $\mathbf{1 0 0 , 0}$ & $\mathbf{1 0 0 , 0}$ & \\
\hline
\end{tabular}

En esta variable se aprecia que los mayores porcentajes de todos estudiantes encuestados se encuentran en el nivel bueno con un 56,67 \%.

\section{Habilidad-técnica}

En la Tabla 3 la dimensión habilidades técnicas de la variable habilidades gerenciales, de 210 estudiantes que representan el 100\% se evidencia que 6 estudiantes que representa el 2,9 $\%$ se encuentran en el nivel deficiente. Asimismo, 83 estudiantes que representa $39,5 \%$ se encuentran en un nivel regular. También 121 estudiantes que representa $57,6 \%$ se encuentran en un nivel bueno.

Tabla 3. Habilidad-Técnica.

\begin{tabular}{llrrrr}
\hline & Frecuencia & Porcentaje & Porcentaje válido & Porcentaje acumulado \\
\hline Válido & Deficiente & 6 & 2,9 & 2,9 & 2,9 \\
& Regular & 83 & 39,5 & 39,5 & 42,4 \\
& Buena & 121 & 57,6 & 57,6 & 100,0 \\
& Total & $\mathbf{2 1 0}$ & $\mathbf{1 0 0 , 0}$ & $\mathbf{1 0 0 , 0}$ & \\
\hline
\end{tabular}


En esta dimensión se aprecia que los mayores porcentajes de todos los estudiantes encuestados se encuentran en el nivel bueno con un 57,62 \%.

\section{Habilidades de trato personal}

En la Tabla 4 la dimensión habilidades de trato personal de la variable habilidades gerenciales, de 210 estudiantes que representan el $100 \%$ se evidencia que 4 estudiantes que representa el 1,9 $\%$ se encuentran en el nivel deficiente. Asimismo, 98 estudiantes que representa $46,7 \%$ se encuentran en un nivel regular. También 108 estudiantes que representa $51,4 \%$ se encuentran en un nivel bueno.

Tabla 4. Habilidades de trato personal.

\begin{tabular}{llrrrr}
\hline & Frecuencia & Porcentaje & Porcentaje válido & Porcentaje acumulado \\
\hline Válido & Deficiente & 4 & 1,9 & 1,9 & 1,9 \\
& & & 46,7 & 46,7 & 48,6 \\
& Regular & 98 & 51,4 & 51,4 & 100,0 \\
& Buena & 108 & $\mathbf{1 0 0 , 0}$ &
\end{tabular}

En esta dimensión se evidencia que los mayores porcentajes de todos los estudiantes encuestados se encuentran en el nivel bueno con un 51,43\%.

\section{Habilidades-conceptuales}

En la Tabla 5 la dimensión habilidades conceptuales de la variable habilidades gerenciales, de 210 estudiantes que representan el $100 \%$ se evidencia que 21 estudiantes que representa el 10 $\%$ se encuentran en el nivel deficiente. Asimismo, 83 estudiantes que representa $39,5 \%$ se encuentran en un nivel regular. También 106 estudiantes que representa $50,5 \%$ se encuentran en un nivel bueno.

Tabla 5. Habilidades-Conceptuales.

\begin{tabular}{llrrrr}
\hline & Frecuencia & Porcentaje & Porcentaje válido & Porcentaje acumulado \\
\hline Válido & Deficiente & 21 & 10,0 & 10,0 & 10,0 \\
& & & 39,5 & 39,5 & 49,5 \\
& Regular & 106 & 50,5 & 50,5 & 100,0 \\
& Buena & $\mathbf{1 0 0 , 0}$ & $\mathbf{1 0 0 , 0}$ & \\
Total & $\mathbf{2 1 0}$ & $\mathbf{1 0 0 , 0}$
\end{tabular}


En esta dimensión se evidencia que los mayores porcentajes de todos los estudiantes encuestados se encuentran en el nivel bueno con un 50,48 \%. Seguido del nivel regular con un 39,52\% y del nivel deficiente con $10,00 \%$.

\section{Discusión}

Los resultados obtenidos en el presente estudio proporcionan información sobre habilidades gerenciales en estudiantes universitarios. En el cual, se evidencia el predominio del nivel bueno con 56,7 $\%$. Es decir, 119 de 210 se encuentran en este nivel. Seguido del nivel regular con 87 estudiantes que representa $41,4 \%$. Luego, el nivel deficiente con 4 estudiantes que representa $1,9 \%$. Se deduce que la mayoría considera las habilidades gerenciales en el proceso de su formación académica. Estos resultados difieren de otro estudio. En el cual, determinan la ausencia de las habilidades gerenciales para una cultura de innovación universitaria en instituciones de educación superior de Colombia y Venezuela (Donawa y Gámez, 2019).

Por otro lado se ha evidenciado que contar con habilidades gerenciales será posible crear una planeación estratégica adecuada en las Pymes que ayude a la gerencia a determinar las acciones que deberá llevar a cabo para lograr el objetivo o la meta de la organización, es decir, le dará la ruta a seguir para la realización de sus actividades y poder llegar a lograr competitividad empresarial (Leyva et al., 2018). En este sentido, se deduce que el abordaje de las habilidades gerenciales en estudiantes de Administración asegura la competitividad empresarial.

En otro estudio se ha observado que las habilidades gerenciales influyen en la efectividad de las agro empresas del valle de Mexicali y, por lo tanto, en su competitividad (González y Ley, 2019). Asimismo, se han demostrado que la planeación estratégica y las habilidades gerenciales como factores internos de las Pymes influyen en la competitividad empresarial $(\mathrm{H} 1: \beta=-0.133$; $\mathrm{p}<$ 0.05) (Leyva et al., 2018).

De igual forma, los resultados obtenidos, en la dimensión habilidades técnicas de la variable habilidades gerenciales se evidencian el predominio del nivel bueno con 57,6\%. Es decir, 121 estudiantes de 210 se encuentran en este nivel. Seguido del nivel regular con 39,5\%. También del nivel deficiente con 2,9\%. Estos resultados difieren de otro estudio. En el cual, se puede observar que las habilidades técnicas con las medias más bajas son: Administración efectiva del tiempo, que permite el logro de los objetivos de la empresa de acuerdo con el plan de calidad propuesto; Manejo del estrés, que tiene consecuencias negativas en el rendimiento de los gerentes; y Conocimiento de software y herramientas informáticas, y su utilización efectiva en el análisis de la información de datos estadísticos, que en forma integral se miden en términos de efectividad gerencial, las cuales se requieren desarrollar ampliamente en los gerentes (Rodriguez et al., 2017).

\section{CONCLUSIONES}

En conclusión, se ha evidenciado que la mayoría de los estudiantes de Administración se encuentra en un nivel bueno de habilidades gerenciales. La minoría en el nivel deficiente y regular. Además, en la dimensión habilidades técnicas se han demostrado que el 57,6\% se encuentra en un nivel bueno. Asimismo, en la dimensión habilidades de trato personal el $51,4 \%$ se encuentra en un nivel bueno. También, en la dimensión habilidades conceptuales, el $50,5 \%$ se encuentra en el nivel Bueno.

Se recomienda realizar estudios de tipo aplicado sobre las habilidades gerenciales con estudiantes de Administración. 
REFERENCIAS

Carhuancho, I., Sicheri, L., Nolazco, F., y De la Torre, J. (2019). Estandarización del cuestionario para medir las habilidades gerenciales en el sector Turismo (HGT). INNOVA Research Journal, 4(3), 1-12. https://doi.org/10.33890/innova. v4.n3.2019.1013

Cassab, P., y Mayorca, D. (2018). Habilidades gerenciales en mujeres comerciantes cabeza de hogar y economía familiar. Revista Escuela de Administración de Negocios, 84, 153166. https://doi.org/10.21158/01208160. n84.2018.1922

Donawa, Z., y Gámez, W. (2019). Ausencia de habilidades gerenciales para una cultura de innovación universitaria en instituciones de Colombia y Venezuela. Universidad y Empresa, 21(36), 8. https://doi.org/10.12804/revistas. urosario.edu.co/empresa/a.5961

González, M. R., y Ley, J. (2019). Habilidades gerenciales y su influencia en la competitividad de las agroempresas del valle de Mexicali, México. Revista Espacios, 40(42), 16.

Hellriegel, D., Jackson, S., y Slocum, J. (2017). A dministración

Hernández, R., y Mendoza, C. (2018). Las rutas Cuantitativa Cualitativa y Mixta. In Metodología de la investigación. Las rutas cuantitativa, cualitativa y mixta

Koontz, H., Weihrich, H., y Cannice, M. (2012). Administración una perspectiva global $y$ empresarial (McGRAW-HILL/ (ed.))
Leyva-Cárdenas, A. B., Espejel-Blanco, J. E., y Cavazos-Arroyo, J. (2017). Habilidades gerenciales como estrategia de competitividad empresarial enlas pequeñas y medianas empresas (Pymes). Revista Perspectiva Empresarial, 4(1). https://doi.org/10.16967/rpe.v4n1a1

Leyva, A., Cavazos, J., y Espejel, J. (2018). Influencia de la planeación estratégica y habilidades gerenciales como factores internos de la competitividad empresarial de las Pymes. Contaduría y Administración, 63(3), 41. https:// doi.org/10.22201/fca.24488410e.2018.1085

Naranjo, R., y González, M. (2012). Management skills of the leader in id size companies of the Colombian caribbean region. Revista Científica Teorías, Enfoques y Aplicaciones En Las Ciencias Sociales, 5(10), 25-36. http://dialnet.unirioja.es/servlet/articulo? codigo $=4736090$ yinfo $=$ resumenyidioma $=E N G$

Rodriguez, G., Escamilla, M., y Centeno, M. (2017). Habilidades Gerenciales. Igarss 2014, 1, 1-5

Whetten, D., y Cameron, K. (2005). Derarrollo de habilidades directivas. In The effects of brief mindfulness intervention on acute pain experience: An examination of individual difference (sexta edic, Vol. 1)

Whetten, D., y Cameron, K. (2011). Desarrollo de habilidades directivas 